\title{
Effects of the advance ratio on the Evolution of Propeller wake
}

\author{
D. G. Baek, J. H. Jung \\ Department of Naval Architecture and Ocean Engineering \\ Pusan National University \\ San 30, Jangjeon 2-Dong, Gumjeong-Gu \\ Busan 609-735, Korea
}

\author{
H. S. Yoon \\ Global Core Research Center for Ships and Offshore Plants \\ Pusan National University \\ San 30, Jangjeon 2-Dong, Gumjeong-Gu \\ Busan 609-735, Korea
}

Received: December 20, 2020. Revised: March 1, 2021. Accepted: March 12, 2021. Published: March 23, 2021.

\begin{abstract}
This study numerically carried out the propeller open water test (POW) by solving Navier-Stokes equations governing the three-dimensional unsteady incompressible viscous flow with the turbulence closure model of the K- $\omega$ SST model. Numerical simulations were performed at wide range of advance ratios. A great difference of velocity magnitude between the inner region and the outer region of the slipstream tube forms the thick and large velocity gradient which originates from the propeller tip and develops along the downstream. Eventually, the strong shear layer appears and plays the role of the slipstream boundary. As the advance ratio increases, the vortical structures originated from the propeller tips quickly decay. The contraction of the vortices trace is considerable with decreasing the advance ratio.
\end{abstract}

Keywords—-propeller; wake; tip vortex; slipstream; advance ratio; KP505

\section{INTRODUCTION}

As marine vehicles become larger and faster, the loading on their propeller blades increases. This increased propeller loading may lead to problems such as noise, hull vibration, and cavitation at high speed. The geometry of a propeller should therefore be designed to minimize them. In general, modern propeller blades have a complicated geometry, making the wake behind the propellers complicated too. Therefore, any serious attempt to optimize the geometrical shape of modern propellers will require a reliable wake analysis based on detailed experimental measurements.

The flow field analysis around a propeller is complicated by many factors as unsteadiness, three-dimensionality, and high turbulence levels. These properties have been pointed out in many previous experiments such as Laser Doppler Velocimetry (LDV) measurements and PIV measurements. Stella et al. (1998) measured the axial velocity component of a propeller wake, and Chesnaks and Jessup, (1998) investigated the tip vortex flow using LDV. Cotroni et al. (2000) have used PIV and particle tracking velocimetry (PTV), respectively, to investigate the near-wake of an isolated marine propeller in longitudinal planes. Calcagno et al. (2002) investigated the complicated 3-D flow behind a marine propeller in the transverse and longitudinal planes using a stereoscopic PIV
(SPIV) technique. Lee et al. (2004) have compared the flow structures of the same marine propeller for the cases of open free surface and closed surface flows at a rather low Reynolds number.

Recently, due to the improvements of computer performances, Reynolds Averaged Navier Stokes (RANS) solvers are becoming the practical tool. Abdel-Maksoud et al. (1998) investigated viscous flow simulations for conventional and high skew marine propellers. Chen and Stern (1999) evaluated computational fluid dynamics of four-quadrant marine-proposer flow. Watanabe et al. (2003) examined simulation of steady and unsteady cavitation on a marine propeller using a RANS code. Rhee and Joshi (2005) estimated computational validation for flow around marine propeller using unstructured mesh based Navier-Stokes solver. Kawamura et al. (2006) investigated Simulation of unsteady cavitating flow around marine propeller using a RANS CFD code. Mitja Morgut and Enrico Nobile (2009) evaluated Comparison of Hexa-Structured and Hybrid-Unstructured Meshing Approaches for Numerical Prediction of the Flow around Marine Propellers.

As authors' literature survey, there is a few research that provided correlations between the vortical structures and wake in obedience to advance ratio. Therefore, the present study focuses on the propeller induced flow structures such as of the propeller slipstream and the tip vortex according to the advance ratio.

TABLE I. PRINCIPAL PARTICULARS OF KP505

\begin{tabular}{|c|c|}
\hline KP505 & Principal \\
\hline Scale Ratio & 31.6 \\
\hline Diameter, D(m) & 0.250 \\
\hline Pitch/Diameter mean & 0.950 \\
\hline Ae/A0 & 0.800 \\
\hline Hub ratio & 0.180 \\
\hline No. of Blades & 5 \\
\hline Section & NACA66 \\
\hline
\end{tabular}




\section{NUMERICAL DETAILS}

\section{A. Governing equations}

Ship hydrodynamic problems are generally solved with the numerical code in the framework of the Reynolds-Averaged Navier-Stokes (RANS) equations. The continuity equation is

$$
\frac{\partial \rho}{\partial t}+\nabla(\rho v)=0
$$

and the momentum equations are

$$
\frac{\partial}{\partial t}(\rho v)+\nabla(\rho v v)=-\nabla p+\nabla(\bar{\tau})+\rho g+F
$$

where $p$ is the static pressure, $\bar{\tau}$ is the stress tensor, and $\rho g$ and $F$ are the gravitational body force and external body forces (e.g., that arise from interaction with the dispersed phase), respectively, $F$ also contains other model-dependent source terms such as porous-media and user-defined sources.

In the Reynolds averaging, the dependent variables in the instantaneous (exact) Navier-Stokes equations are decomposed into the mean (ensemble-averaged or timeaveraged) and fluctuating components. So the Eqs.(1) and (2) can be written in Cartesian tensor form as

$$
\begin{gathered}
\frac{\partial \rho}{\partial t}+\frac{\partial}{\partial x_{i}}\left(\rho u_{i}\right)=0 \\
\frac{\partial}{\partial t}\left(\rho u_{i}\right)+\frac{\partial}{\partial x_{j}}\left(\rho u_{i} u_{j}\right)= \\
-\frac{\partial p}{\partial x_{i}}+\frac{\partial}{\partial x_{j}}\left[\mu\left(\frac{\partial u_{i}}{x_{j}}+\frac{\partial u_{j}}{\partial x_{i}}-\frac{2}{3} \delta_{i j} \frac{\partial u_{l}}{\partial x_{l}}\right)\right]+\frac{\partial}{\partial x_{j}}\left(-\rho \overline{u_{i}^{\prime} u_{j}^{\prime}}\right)
\end{gathered}
$$

where $\delta_{i j}$ is the Kronecker delta and $-\rho \overline{u_{i}^{\prime} u_{j}^{\prime}}$ are the unknown Reynolds stresses

$$
-\rho \overline{u_{i}^{\prime} u_{j}^{\prime}}=\mu_{t}\left(\frac{\partial u_{i}}{x_{j}}+\frac{\partial u_{j}}{\partial x_{i}}\right)-\frac{2}{3}\left(\rho k+\mu_{t} \frac{\partial u_{l}}{\partial x_{l}}\right) \delta_{i j}
$$

The equations are made closed with the turbulence model, and here the $k-\omega S S T$ model is employed:

$$
\begin{gathered}
\frac{\partial}{\partial t}(\rho k)+\frac{\partial}{\partial x_{i}}\left(\rho k u_{i}\right)=\frac{\partial}{\partial x_{j}}\left(\Gamma_{k} \frac{\partial k}{\partial x_{j}}\right)+\tilde{G}_{k}-Y_{k}+S_{k} \\
\frac{\partial}{\partial t}(\rho \omega)+\frac{\partial}{\partial x_{i}}\left(\rho \omega u_{i}\right)=\frac{\partial}{\partial x_{j}}\left(\Gamma_{\omega} \frac{\partial \omega}{\partial x_{j}}\right)+\tilde{G}_{\omega}-Y_{\omega}+D_{\omega}+S_{\omega}
\end{gathered}
$$

In these equations, $G_{k}$ represents the generation of turbulence kinetic energy due to mean velocity gradients, $G_{\omega}$ the generation of $\omega, \Gamma_{k}$ and $\Gamma_{\omega}$ the effective diffusivities of $k$ and $\omega$, respectively, $Y_{k}$ and $Y_{\omega}$ the dissipations of $k$ and $\omega$ due to turbulence, $D_{\omega}$ the cross-diffusion term, and $S_{k}$ and $S_{\omega}$ the user-defined source terms.

\section{B. Numerical methods}

The RANS formulations are used and equations are solved in a sliding interface method used for unsteady-flow mode. The pressure-velocity coupling and the overall solution procedure are based on the SIMPLEC algorithm. The secondorder scheme is used for pressure, convection terms and second-order central difference scheme for diffusion terms.

\section{Computational schemes}

In the case of unsteady simulation, the whole domain should be computed with the sliding mesh technique. The computational domain is defined with a cylinder of $8.6 \mathrm{D}$ diameter surrounding the propeller and hub. The inlet and outlet boundaries are located at 2.4D upstream and 5.3D downstream the center of the propeller respectively. The domain is split into global stationary part and moving part which is specified by a smaller cylinder enclosing the blades and hub entirely. Trimmer mesh is employed for the global stationary block.

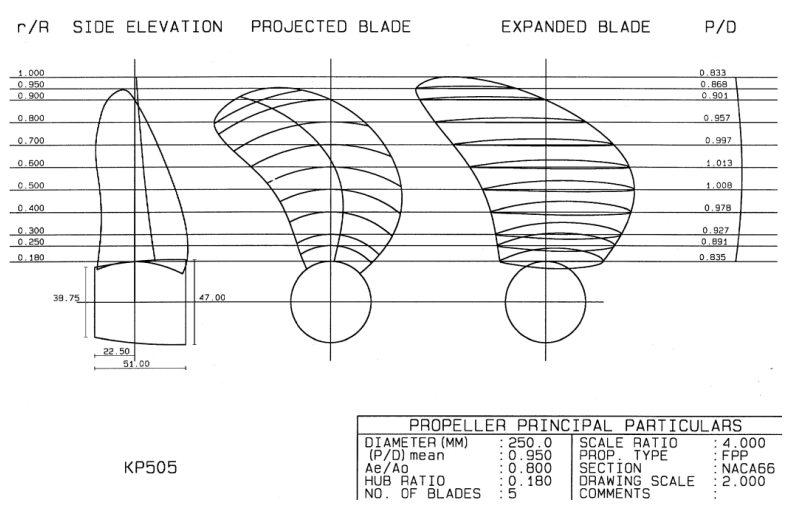

Fig. 1. Geometry of KP505
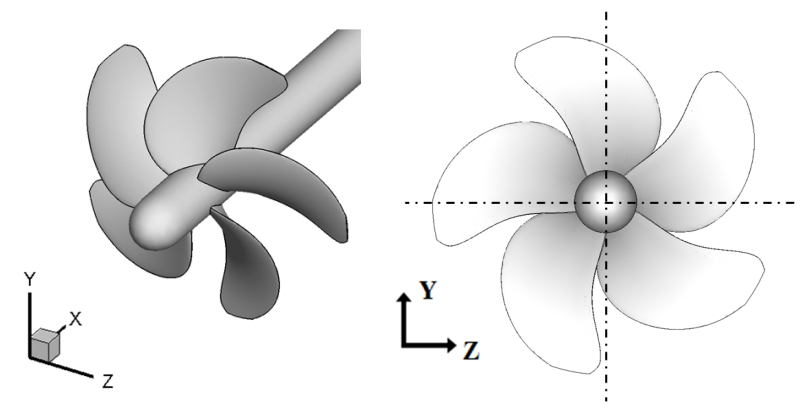

Fig. 2. Coordinate system of KP505 


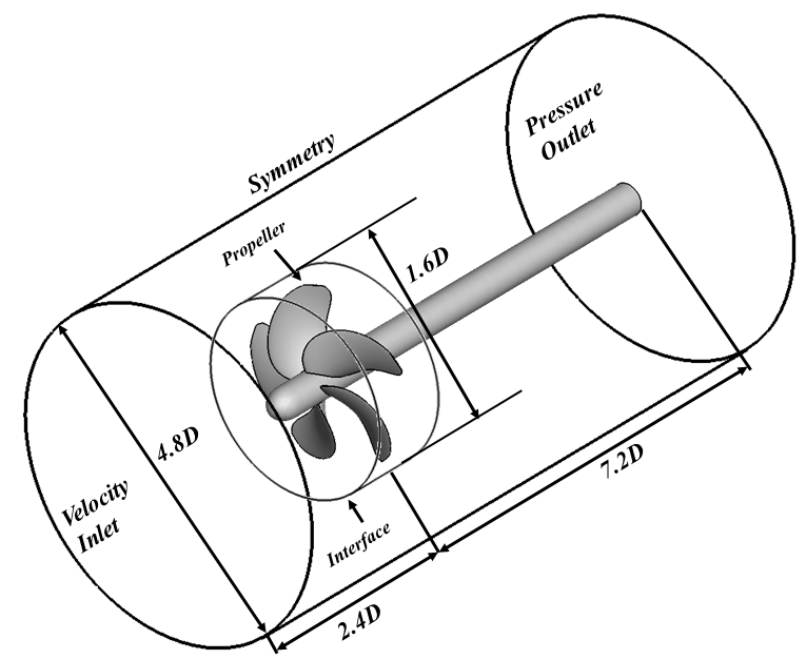

Fig. 3. Schematic of the computational domain and boundary conditions.

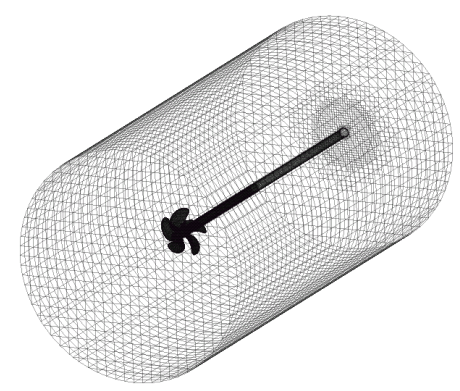

(a)

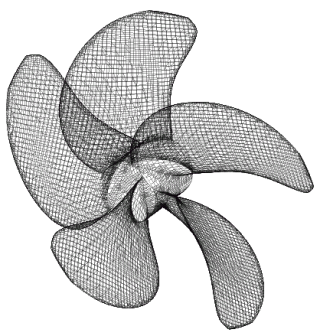

(b)
Fig. 4. Generated grid of (a) overview and (b) propeller around

\section{Validation}

The computed and measured open water data are compared in Fig.6, in which three groups of computed $K_{T}$, $K_{Q}$ and $\eta_{0}$ are included. The results for propeller performance is similar with the experimental results (Fujisawa et al. 2000) especially at smaller $\mathrm{J}$ values, such as at $\mathrm{J}=$ $0.2 \sim 0.5$ with the differences of smaller than $7 \%$ for $K_{Q}$ and at all $\mathrm{J}$ values the differences are smaller than $5 \%$ for $K_{T}$.

As $J$ grows larger, the difference for performance becomes larger. The reason of the big differences may partly be due to the very small absolute value of performance and the doubted experimental results at large values of $J$ may be also considered.

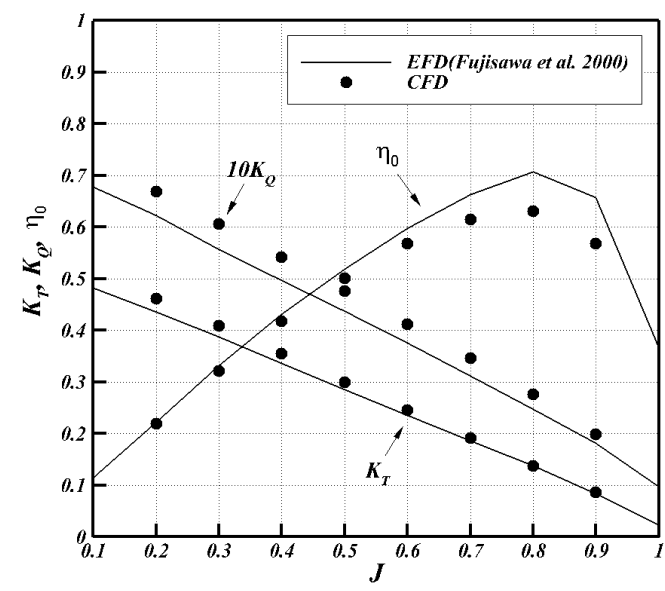

Fig. 5. Validation of open water test and difference

\section{RESULTS AND DISCUSSION}

The typical instantaneous velocity vectors and the axial velocity contours in the plane normal to the propeller plane at phase angle $\Phi=0$ are plottd in Fig. 6 where representatively two advance ratios of $\mathrm{J}=0.2$ and 0.7 are considered. The propeller axis is aligned with $\mathrm{y} / \mathrm{D}=0$, and the propeller plane locates at $x / D=0$. In genreal, the flow behind the propeller is composed of the slipstream tube and the tip vortices, which is more clearly identified with decreasing the advance ratio. At $\mathrm{J}=0.2$ which is the smallest advance ratio or the heaviest load condition among the the advance ratios considered in this study, the freestream and the propeller induced flow superpose, forming the slipstream with the relatively strong flow with large velocity vectors forms within the propeller tip out of which the frestream velocity is predominent to the flow, as shown in Fig. 6(a).

This strafication of the flow is clarified by the contours of the axial velocity in Fig. 6(b). The velocity magnitude in the slipstream tube is much larger than that ouside of the slipstream. This big difference of velocity magnitude between the inside and the out side of the slipstream tube forms the thick and large velocity gradient which originates from the propeller tip and develops along the downstream. Eventually, the strong shear layer appears and play the role of the slipstream boundary.

As the advance ratio increases, the propeller load becomes light. Therefore, the difference of velocity magnitude between the slipstream and the outside becomes minor, resulting in almost disappearance of the shear layer which clearly appeared at lower advance ratio of $\mathrm{J}=0.2$ in Figs. 6(a) and 6(b). These variations of the wake according to the increases of the advance ratio are clarified by the velocity vectors and the axial velocity contours as shown in Figs. 5(c) and 5(d), respectively. 

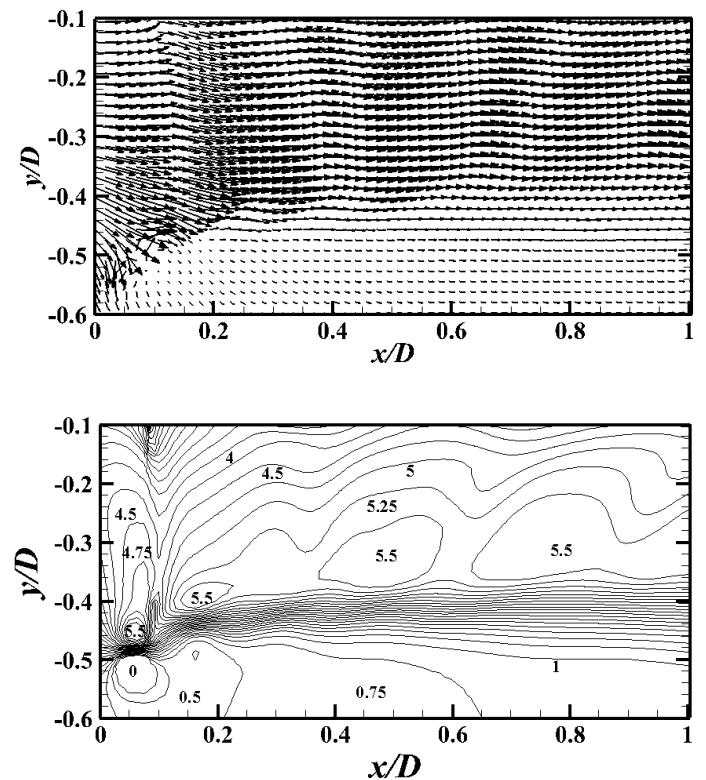

(a)
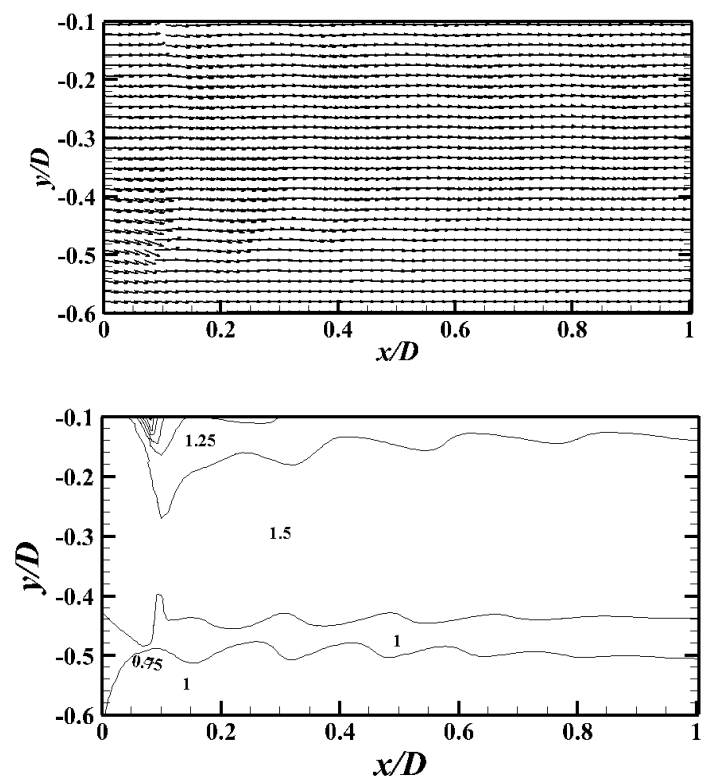

(b)

Fig. 6. Typical instantaneous velocity vector fields and axil velocity contour in the longitudinal plane at $\phi=0^{\circ}$ (a) $\mathrm{J}=0.2$, (b) $\mathrm{J}=0.7$.

The other component of the near wake of the propeller is the tip vortices which are shed successively from the tip of each blade with a regular interval. Paik et al. (2007) used the Galilean decomposition method to understand the coherent vortex structure of the wake behind a rotating propeller. Thus, the present study also adopts the Galilean decomposition method to identify the regular appearance of the tip vortices along the downstream behind of the propeller.

Fig. 7 shows the appropriately decomposed instantaneous velocity field for two advance ratios of $\mathrm{J}=0.2$ and 0.7 . The several different convection velocities as the translational velocity of vortices are considered to obtain the proper value of the convection velocity $(\mathrm{Uc})$ which is subtracted from the axial component of the instantaneous velocity as already shown in Fig. 6 to detect the rotational flow corresponding to the tip vortices. Eventually, in the present study, $\mathrm{Uc}=1.2 \mathrm{U} 0$ is adapted to the Galilean decomposition.
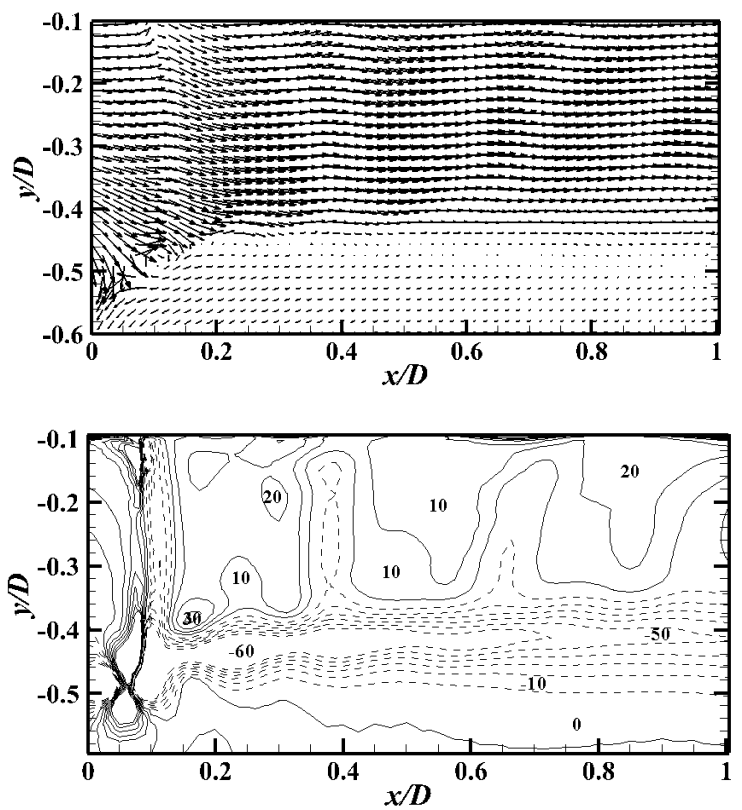

(a)
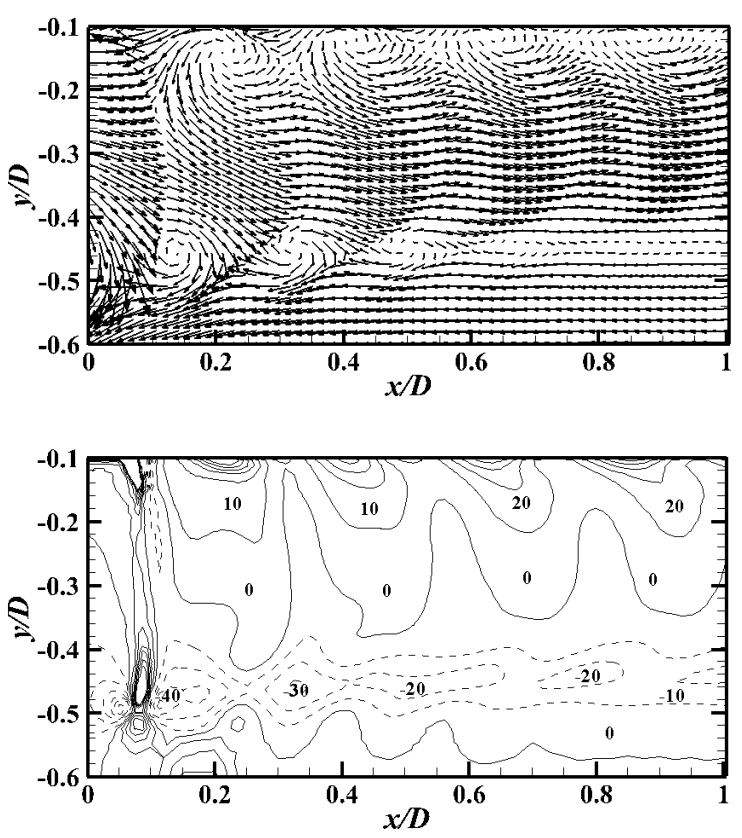

(b)

Fig. 7. Contour of vorticity(left) and Typical instantaneous velocity vector fields subtracted by convection velocity $\left(\boldsymbol{U}_{\boldsymbol{c}}=1.2 \boldsymbol{U}_{\boldsymbol{0}}\right)$ of $\mathrm{J}=0.2$ and $\mathrm{J}=0.7$ at the phase angle $\phi=0^{\circ}$ 


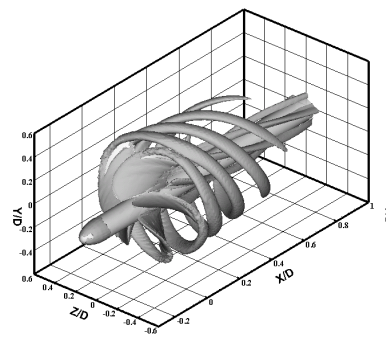

(a)

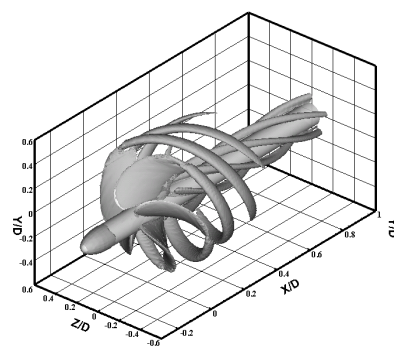

(b)

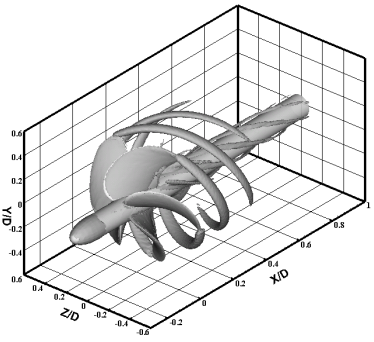

(c)

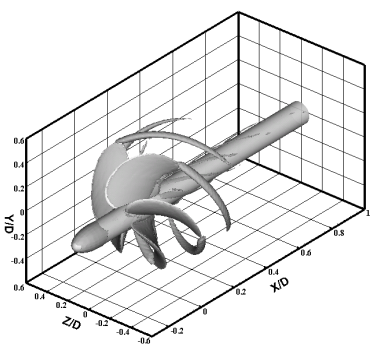

(d)
Fig. 8. KP505 iso-surface of $\mathbf{L a m d a 2}=-5,000$ for four different loading conditions during transient acceleration (a) $\mathrm{J}=0.2$, (b) $\mathrm{J}=0.7$.

At both advance ratios of $\mathrm{J}=0.2$ and $\mathrm{J}=0.7$, the rotational flow motion appears near the tip and a large velocity gradient occurs in the wake sheet. The convection velocity of the wake sheet is larger than that of tip vortices in the slipstream region. Especially, at lower advance ratio, the convection velocity of the wake sheet is much larger than that of tip vortices in the slipstream region.

Successively, the rotational flow motion in the further downstream is not clearly captured as shown in Fig. 7(a), even though the Galilean decomposition is used to detect the rotational flow. Consequently, the large difference of the convective velocity between the tip vortices and the wake sheet contribute to the distinct formation of the shear layer and the spatial evolution of vortical structure. It could be confirmed with vorticity contour as shown in Fig.7 (a). In conformity with the preceding, the vorticity from the tips elongated and moved downstream. As the load condition becomes light, vorticity contour has a strong asymmetry shape like an oval with a short minor axis. The asymmetry of the vorticity was caused by the interaction between the tip vortex and the wake sheet as shown in Fig. 7(b). The present results of the formation of the rotational flow motion and the wake sheet are consistent with the findings of Paik et al. (2007).

In order to define three-dimensional vortical structures originated from the propeller tip, we adopted the method given by Jeong \& Hussain(1995), who defined a vortical region as the region with negative, the second largest eigenvalue of, where and are the strain-rate tensor and rotation-rate tensor, respectively.

As the advance ratio increases or the load condition becomes light, the vortical structures originated from the propeller tips quickly decay, as shown in Fig. 8. This result is supported by the velocity vectors and the axial velocity contours in Figs. 6 and 7 which showed that the shear layer formed by the large difference of the velocity gradient between the slipstream and the outside of the slipstream becomes stronger with decreasing the advance ratio.
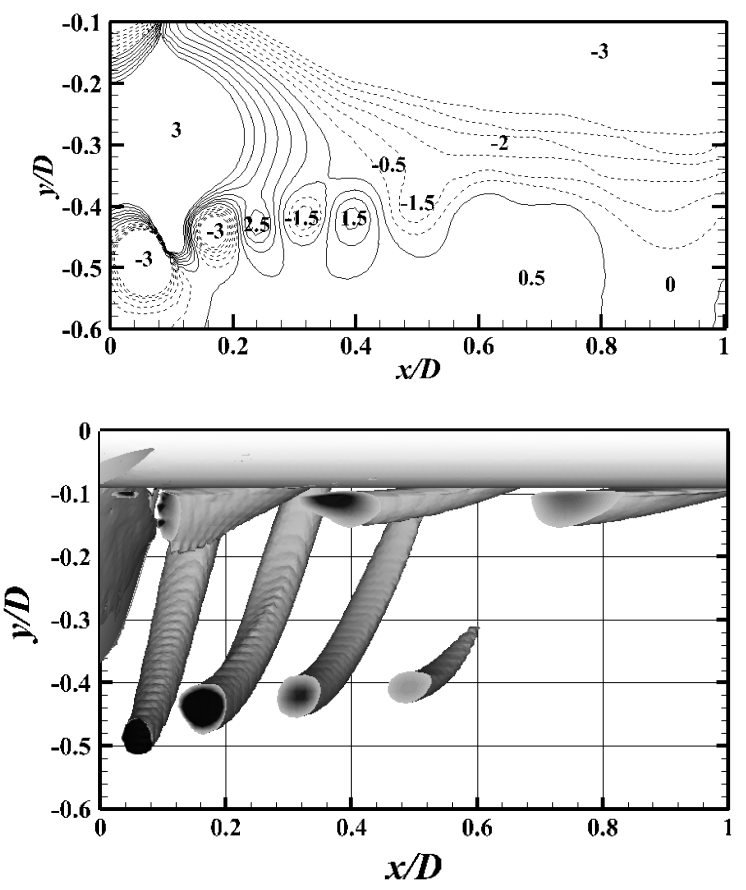

(a)
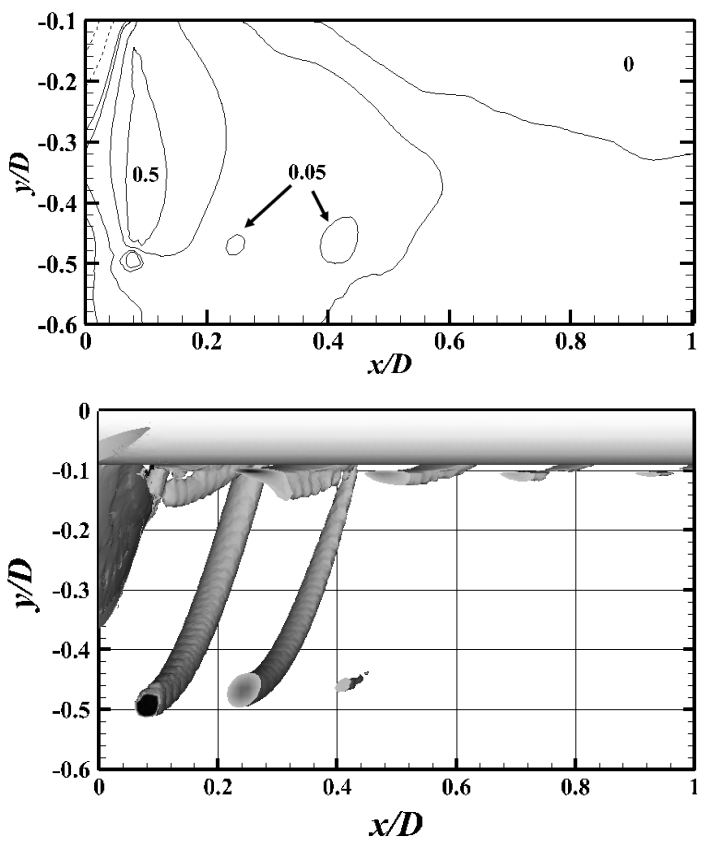

(b)

Fig. 9. Contour of pressure coeffcient in the longitudinal plane at $\Phi=0^{\circ}$. (a) $\mathrm{J}=0.2$, (b) $\mathrm{J}=0.7$

Figure 9 shows the isosurface of and the contours of pressure coefficients in $\mathrm{x}-\mathrm{y}$ plane at $\Phi=0$ for $\mathrm{J}=0.2$ and $\mathrm{J}=0.7$. As early shown in Fig. 8, the heavy load condition of $\mathrm{J}=0.2$ 
sustains the vortical structures originated from each tip to further downstream, compared to the case of light load condition of $\mathrm{J}=0.7$, which can be clarified by comparing between Fig. 9(a) and Fig. 9(b) for $\mathrm{J}=0.2$ and $\mathrm{J}=0.7$, respectively.

At the heavy load condition of $\mathrm{J}=0.2$, the strong shear layer, which is derived by big difference of velocity magnitude between the inside and the outside of the slipstream tube as early observed in Figs. 6(a) and 6(b), contributes to the addition of the rotational motion to the tip vortices and eventually the long survival of the propeller tip vortices to farther downstream. Thus, as the advance ratio decreases, the lower pressure forms at the center of each tip vortices, which can be clarified by comparing between Fig. 9(a) and Fig. 9(b) for $\mathrm{J}=0.2$ and $\mathrm{J}=0.7$, respectively. Additional, in the slipstream region, the pressure becomes much lower with decreasing the advance ratio.

The traces of the tip vortices shed from each propeller tip are plotted in Fig. 10 where the location of the tip vortex is identified by the center of the tip vortex (maximum vorticity, the place of the lowest pressure) as observed in Fig. 8. As the advance ratio decreases, the contraction of the trace is considerable. Especially, the slope of the contraction is rapid near wake region owing to the stronger interaction between the tip vortices with higher rotational energy, regardless of the advance ratio. Then the trace becomes saturated earlier with increasing the advance ratio.

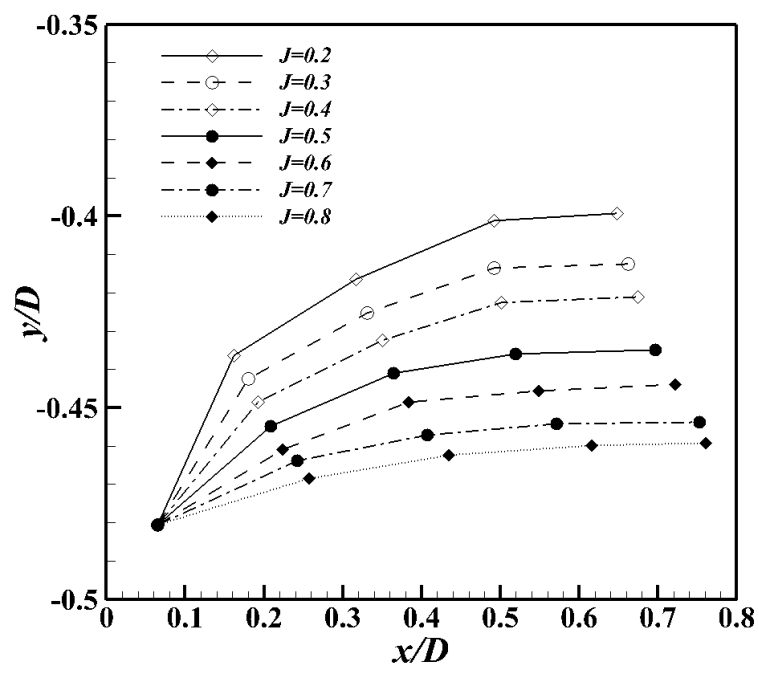

Fig. 10. Location of tip vortices on the $\mathrm{X}-\mathrm{Y}$ plane and contraction rates(right) for different advance ratio of $\mathrm{J}=0.2 \sim 0.8$

\section{CONCLUSIONS}

This study numerically carried out the propeller open water test (POW) by solving Navier-Stokes equations governing the three-dimensional unsteady incompressible viscous flow with the turbulence closure model of the K- $\omega$ SST model. Numerical simulations were performed at wide range of advance ratios. A great difference of velocity magnitude between the inner region and the outer region of the slipstream tube forms the thick and large velocity gradient which originates from the propeller tip and develops along the downstream. Eventually, the strong shear layer appears and plays the role of the slipstream boundary. As the advance ratio increases, the vortical structures originated from the propeller tips quickly decay. The contraction of the vortices trace is considerable with decreasing the advance ratio.

\section{ACKNOWLEDGMENT (Heading 5)}

This work was supported by National Research Foundation of Korea (NRF) grant founded by the Korea government (MSIP) through GCRC-SOP (No. 2011-0030013).

Also, this work was supported by the Technology Innovation Program (10033689, Technology development of propeller and rudder for a ship with low vibration and high efficiency) funded by the Ministry of Knowledge Economy (MKE, Korea)

\section{REFERENCES}

[1] Jeong, J. \& Hussain, F.(1995). On the identification of a vortex, Journal of Fluid Mehanics, vol. 285, pp. 69-94.

[2] Abdel-Maksoud, M., Menter, F., and Wuttke, H. (1998). Viscous flow simulations for conventional and highskew marine propellers. Ship Technology Research, 45:64 - 71.

[3] Stella A, Guj G, Di Felice F, Elefante M (1998). Propeller wake evolution analysis by LDV. Proc of 22nd Symposium on Naval Hydrodynamics, Washington D.C., pp 171-188

[4] Chesnaks C, Jessup S (1998). Experimental characterisation of propeller tip flow. Proc of 22nd Symposium on Naval Hydrodynamics, Washington D.C., pp 156-169

[5] Chen, B. and Stern, F. (1999). Computational fluid dynamics of fourquadrant marine-propulsor flow. Journal of Ship Research, 43(4):218 228.

[6] Cotroni A, Di Felice F, Romano GP, Elefante M (2000). Investigation of the near wake of a propeller using particle image velocimetry. Exp Fluids 29:S227-236

[7] Calcagno G, Di Felice F, Felli M, Pereira F (2002). Propeller wake analysis behind a ship by stereo PIV. Proc of 24th Symposium on Naval Hydrodynamics, Fukuoka, 3:112-127

[8] Watanabe, T., Kawamura, T., Takekoshi, Y., Maeda, M., and Rhee, S. H. (2003). Simulation of steady and unsteady cavitation on a marine propeller using a rans cfd code. In Fifth International Symposium on Cavitation, CAV2003, Osaka, Japan

[9] Sang Joon Lee, Bu Geun Paik, Jong Hwan Yoon, Choung Mook Lee(2004), Three-component velocity field measurements of propeller wake using a stereoscopic PIV technique, Experiments in Fluids 36, 575-585

[10] Rhee, S. H. and Joshi, S. (2005). 'Computational validation for flow around marine propeller using unstructured mesh based navier-stokes solver'. JSME International Journal, Series B, 48(3):562 - 570.

[11] Kawamura, T., Takekoshi, Y., Yamaguchi, H., Minowa, T., Maeda, M., Fujii, A., Kimura, K., and Taketani, T. (2006). 'Simulation of unsteady cavitating flow around marine propeller using a rans cfd code'. In Sixth International Symposium on Cavitation, CAV2006, Wageningen, The Netherlands.

[12] Bu-Geun Paik, Jin Kim, Young-Ha Park, Ki-Sup Kim, Kwon-Kyu $\mathrm{Yu}(2007)$. Analysis of wake behind a rotating propeller using PIV technique in a cavitation tunnel, Ocean Engineering (34)

[13] Mitja Morgut, Enrico Nobile(2009). Comparison of Hexa-Structured and Hybrid-Unstructured Meshing Approaches for Numerical Prediction of the Flow Around Marine Propellers. First International Symposium on Marine Propulsors smp'09, Trondheim, Norway

\section{Creative Commons Attribution License 4.0 (Attribution 4.0 International, CC BY 4.0)}

This article is published under the terms of the Creative Commons Attribution License 4.0 https://creativecommons.org/licenses/by/4.0/deed.en_US 